\title{
Análisis del Enfoque de Evaluación Institucional aplicado por la Gestión Educativa de Costa Rica Christian School
}

\author{
Analysis of the institutional evaluation approach applied to the educational \\ management of Costa Rica Christian School
}

\author{
Ana Jenssie Campos Campos \\ Costa Rica Christian School, Costa Rica. \\ anajcampos@gmail.com
}

Recibido: 25 abril 2011 Aceptado: 25 abril 2011 Corregido: 29 junio 2011

\section{Resumen}

El presente artículo corresponde a una investigación sobre el análisis del enfoque de evaluación institucional que aplica la gestión educativa de un centro escolar privado de la Región Educativa de San José Norte. Para el análisis se consideraron como objetivos: identificar el enfoque de evaluación institucional a partir de las características de tres enfoques sobre evaluación institucional, determinar las dimensiones del enfoque utilizado y como tercer objetivo se propuso determinar la percepción que tiene el personal de la institución sobre el nivel de influencia que tiene sobre la organización el enfoque de evaluación institucional empleado.

El sustento teórico del estudio se fundamenta en tres enfoques de evaluación institucional: a saber: evaluación del currículo, desde el paradigma cualitativo, cuyos expositores son: Posner, Eisner y Stake, mencionados por Flórez (2003), evaluación CIPP (Contexto, Entrada, Proceso y Producto) de Stufflebeam y Shinkifield citados por Bausela (2003), y el enfoque de autoevaluación, Antúnez (2009). La propuesta de intervención se basó en este último enfoque. La metodología utilizada para el estudio fue cuantitativa descriptiva, y se utilizó un cuestionario semiestructurado autoadministrado a la población de la organización objeto de estudio en las áreas: docente, técnico-administrativa, administrativa docente y administrativa. Los resultados que arrojó el estudio, muestran que aunque hay algunas características de los tres enfoques propuestos, en cuanto a la determinación de las dimensiones de la evaluación institucional, existe un enfoque de evaluación institucional específico en el centro en estudio. Por lo anterior, la percepción del personal consultado, en cuanto a la influencia que tiene el enfoque de evaluación aplicado en la institución no es significativa. Desde la función de evaluar que tiene la administración de la educación en cada centro educativo, la gestión de un proceso de esta naturaleza, sin importar el enfoque que se utilice, tiene el objetivo de determinar las necesidades existentes y, con ello, de procurar el logro de las metas de la organización. Esto involucra un proceso de construcción de una cultura de evaluación que no existe en el medio educativo latinoamericano ni nacional. Cuando esta construcción se basa en el enfoque de autoevaluación, 


\title{
Gestión: Gestion
}

Revista Cientifica Digital ISSN-2215-2288

el proceso es participativo, ya que los actores sociales interactúan de manera directa y reflexiva, lo que proporciona compromiso con el proyecto de mejora institucional. Se finaliza con una propuesta de intervención desde la gestión educativa en el área de evaluación.

Palabras clave: Educación, administración de la educación, calidad, evaluación, evaluación institucional, enfoques modélicos.

\begin{abstract}
The following article corresponds to the synthesis of a research work about the analysis of the Institutional Evaluation Approach applied to the educational management of a private school in the San José Norte Educational Region. The objectives of the analysis lied in identifying the institutional evaluation approach from the characteristics of the three approaches proposed, as well as on determining the dimensions of the approach used, and a third objective was determining the staff perception of the institution in terms of the level of impact that the institutional evaluation approach has in regard to the organization.
\end{abstract}

The theoretical support for the study is based on three institutional evaluation approaches, that is, curriculum evaluation, from the qualitative paradigm, whose exponents are: Posner, Eisner and Stake, mentioned by Flórez (2003), Stufflebeam and Shinkifield's CIPP (Context, Input, Processes and Products) evaluation, cited by Bausela (2003), and the self-evaluation approach, Antúnez (2009). The intervention proposal is based on this latter approach. The methodology used for the study is the quantitative-descriptive methodology, and a semistructured and self-applied questionnaire was applied to the organization's population in the area: teaching, technical-administrative, teaching-administrative and administrative staff.

The results produced by the study clearly establish that while it is possible to identify several characteristics of the three approaches proposed, in respect to the determination of the dimensions, it does not establish a specific institutional evaluation approach to be applied by the school educational management. Due to the above, the perception of the participating staff concerning the impact that the evaluation approach applied has on the organization does not represent a significant impact on the organization. From the evaluative duty that educational administration has on each school, the management of an evaluation process will have the objective to determine the existing needs, regardless of the approach used, and also to produce the achievement of the goals proposed by the organization. The above involves an evaluative culture construction process, which currently does not exist either in the Latin American or national educational environment. When this construction is based on the self-evaluation approach, the process becomes participatory, as social actors interact in a direct and reflective 


\section{Gigestión:}

Revista Cientifica Digital ISSN-2215-2288

manner, which brings about commitment towards the institutional improvement process. Finally includes a program to administrate from the evaluation area.

Keywords: Education, education management, quality, evaluation, institutional evaluation, model approaches.

\section{Introducción}

La educación se convirtió en una herramienta indiscutible para vencer los diferentes desafíos que cada época le presenta. En la actualidad no son menos los obstáculos por enfrentar y vencer; al contrario, se muestran de manera más deliberada, como resultado del constante cambio en el orden social, económico y político según la sociedad a la que se pertenece.

Atender los desafíos que generan los avances globales, sobre todo en el ámbito tecnológico, es parte del reto que enfrenta el sistema educativo, en todas sus etapas en cuanto a la formación de las nuevas generaciones de manera que, estas, respondan a las demandas de la sociedad; en la capacidad de aprendizaje, las destrezas laborales y las competencias personales para disputar y ser productivo en medio de una sociedad económicamente demandante.

En la sociedad actual, dentro del sistema educativo y en el área administrativa en general, el concepto de evaluación institucional, ha cobrado un nuevo valor. Desde la administración general se ha filtrado en la gestión educativa la importancia de atender la evaluación institucional, como punto medular para alcanzar mejoras institucionales u organizacionales, en donde la participación de la comunidad es relevante. Delors, Mufti, Amagi, Carneiro, Chung, Geremek y Nanzhao (1996) indica que "La participación de la comunidad local en la evaluación de las necesidades, mediante un diálogo con las autoridades públicas y los grupos interesados dentro de la sociedad, es una primera etapa fundamental para ampliar el acceso a la educación y para mejorarla” (p.26). Esta etapa de diagnóstico es esencial para iniciar un proceso de evaluación institucional que conlleve a la mejora en la calidad de la educación.

La importancia que el presente trabajo representa para la educación costarricense deriva de la significación que a nivel internacional se está brindando a los procesos de evaluación de las organizaciones educativas, con el fin de proporcionar la información necesaria para llevar a cabo los cambios y mejoras a la calidad a través de la toma de decisiones, por otra parte, la evaluación 
institucional es uno de los aspectos institucionales al que el gestor o gestora de la educación debe propiciar con el fin de conocer las principales necesidades de la organización y con ello tomar decisiones que orienten el cambio y mejora en la calidad del servicio educativo.

El proceso de evaluación institucional, como parte de la cultura organizacional proporcionará al administrador o administradora de la educación, la información necesaria para identificar las necesidades e implementar los cambios requeridos, para hacer frente a las debilidades que a través de la reflexión y análisis de las diferentes dimensiones de la organización se manifiesten.

El trabajo de investigación está fundamentado en dos objetivos generales:

1. Analizar las dimensiones del enfoque de evaluación institucional aplicado por la gestión educativa de Costa Rica Christian School.

2. Diseñar una propuesta de evaluación institucional a partir de la gestión de un centro educativo privado, Costa Rica Christian School.

Por medio de los objetivos específicos se pretende:

1.1 Identificar el enfoque de evaluación institucional aplicado por la gestión de un centro educativo privado, Costa Rica Christian School.

1.2 Determinar las dimensiones presentes en el enfoque de evaluación institucional aplicado por la gestión de un centro educativo privado, Costa Rica Christian School.

1.3 Determinar la percepción que el enfoque de evaluación institucional aplicado por la gestión educativa de Costa Rica Christian School ha desarrollado entre el personal de la organización educativa.

2.1 Diseñar una propuesta de evaluación institucional con base al resultado del análisis de las dimensiones del enfoque actual aplicado por la gestión educativa en Costa Rica Christian School. 


\section{Fundamentación teórica}

\subsection{Concepto de Evaluación}

Referirse al término de evaluación implica un proceso de reflexión, sin embargo, por lo general en el ámbito educativo se ha relacionado más con la evaluación de los aprendizajes de los y las estudiantes, o bien con el desempeño del docente; no obstante evaluación institucional de centros educativos involucra otras dimensiones.

El concepto en sí mismo, según Edwards (1991), ha sido muy discutido, por la necesidad de control de procesos con el fin de lograr sistemas eficientes y eficaces. La evaluación puede ser concebida de dos formas; aunque diferentes, relacionadas, como medición (se refiere a la cuantificación de datos, números o puntajes), ésta ha llevado al desarrollo de pruebas y técnicas, cuya información es válida y confiable; y la definida y practicada como determinación de logros comparándolos con los objetivos propuestos, esta otra concepción ubica en el centro de la investigación los logros de un programa educativo en relación con los objetivos propuestos.

Al considerar el concepto de evaluación, Ruiz (2008, p.18), presenta una definición en términos generales, percibiéndola como un "proceso de análisis estructurado y reflexivo, que permite comprender la naturaleza del objeto de estudio y emitir juicios de valor sobre el mismo, proporcionando información para ayudar y ajustar la acción educativa.” Es importante aclarar que, el concepto de evaluación, dista mucho del de medición, que tradicionalmente se ha tomado como semejante. Con referencia a los conceptos de evaluación, Mora (2004) indica que el Joint Committee on Standards for Educational Evaluation señala que ésta es "el enjuiciamiento sistemático de la validez o mérito de un objeto” (p.2).

\subsection{Evaluación de la educación}

Referirse al concepto de evaluación educativa, implica considerar, según Edwards (1991), la práctica de evaluar la calidad de la educación que se proporciona, por lo que es necesario estimar este proceso como una práctica institucionalizada en el sistema educativo, en tanto en que, incide en el fenómeno de adquisición de conocimientos. La evaluación por consiguiente 
como lo comenta Edwards (1991), debe realizarse de forma holística, “considerando la unidad e integridad que caracterizan a los procesos educativos” (p.39).

Por lo anterior, se hace necesario establecer los indicadores que se refieren a una evaluación de la calidad de la educación, algunos de los indicadores que deben ser considerados, según Edwards (1991), son los siguientes

Heterogeneidad cultural y social de los alumnos; diversificación de la educación; flexibilidad de la estructura organizativa para atender la población heterogénea; niña de edad escolar regular; niños desfasados en edad-grado, desertores jóvenes y adultos con primaria incompleta, niños que viven en poblaciones urbano-marginales y rurales entre otros. (pp. 40-41)

Las concepciones más recientes de evaluación de la educación, la ubican en un paradigma menos tradicional, en donde la reflexión y el consenso son parte importante del este proceso , así lo expone González, Nieto y Portela (2007, p. 266), la evaluación como un proceso que permite evaluar el centro escolar se concibe como "una actividad de reflexión, comprensión, valorización y toma de decisiones, es concebida como un proceso transversal a todas y cada una de las actuaciones que ocurren en educación, así como al conjunto de factores, procesos y agentes que participan en la misma.”

Desde cualquier punto de vista que se revisen las definiciones sobre evaluación institucional, el principal objetivo está relacionado con mejorar la condición actual de la organización a través de intervenir oportunamente, en donde se logre disminuir la brecha entre la situación actual y la propuesta de mejora. La evaluación, en el ámbito educativo, está siempre al servicio de los objetivos, y se considera como una función instrumental. Por su finalidad, representa, una doble aportación a la calidad en este campo.

Es evidente entonces, que la cultura institucional conformada por: el cuerpo directivo, personal docente, padres y madres de familia, así como el alumnado, construyen el conjunto de sujetos a los que debe ir dirigido la investigación evaluativa en un centro educativo. 


\subsection{Acercamiento al concepto modelo de evaluación institucional}

En los sistemas educativos latinoamericanos se han adoptado los principios teóricos que fundamentan los diferentes modelos de evaluación, estos se han transformado a través de los periodos y las reformas educativas adoptadas por las políticas implementadas.

La evolución que ha sufrido la evaluación y los principios teóricos de los modelos que la sustentan, ha sido un recorrido entre los paradigmas cuantitativo y cualitativo, en donde la mayor producción de modelos se ubica en el siglo XX, pasando de la medición observable desde el enfoque positivista, hasta la evaluación en donde la participación y el juicio de valor, adquieren un rol importante para el evaluador, así como, para los otros agentes participantes.

Una concepción sobre modelo en evaluación la proporciona, Antúnez y Carnicero (2009), donde establece que es:

...el dispositivo de principios que orientan la intención evaluadora y las indicaciones en relación con los ámbitos de evaluación, con los momentos, las fuentes e instrumentos que se pueden utilizar, los procedimientos y los agentes que intervienen en ellos. Se entiende como los principios del modelo, el conjunto de normas o ideas fundamentales que rigen la propuesta de modelo. (pp. 125-126)

Se entiende en este estudio, que el modelo es aquel proceso sistemático aplicado por la gestión de la organización educativa para valorar y comprender el proceso para la formulación de juicios de valor, con el fin de proporcionar la información requerida para realizar los cambios necesarios a favor de la acción educativa.

Como característica, puede encontrarse en las modalidades de evaluación interna, según Escudero, González y Del Cerro (1998), citado por González et al., (2007, p. 270), que “asumen el carácter contextual, diverso y constructivo de la educación y del ejercicio de la profesión docente.” En este sentido, el proceso de evaluación interna considera el ambiente en que se desenvuelve la organización en el aspecto macro y micro del sistema educativo, y con ello la práctica docente que la lleva a cabo. 


\subsection{Importancia de la evaluación institucional interna}

La evaluación interna es un imperativo de la actividad del centro, no es suficiente el medir la calidad de un centro por las calificaciones de sus alumnos. Para Santos (1995), la evaluación interna resulta importante porque:

- $\quad$ Permite reflexionar sobre lo que se hace.

- Facilita la coordinación vertical y horizontal.

- Ayuda a comprender lo que sucede.

- Impulsa el diálogo y la participación.

- Permite tomar decisiones racionales.

- Impide los solapamientos.

- Ayuda a incidir sobre lo que se considera sustancial.

- Permite corregir errores.

- Ayuda a intensificar el esfuerzo en lo esencial.

- Permite aprender nuevas cosas.

- Hace ganar coherencia al equipo docente.

- Se convierte en un ejemplo para los alumnos.

- Ayuda al perfeccionamiento del profesorado.

Cada una de las razones anteriores, justifican la importancia de realizar un proceso de evaluación interna a partir de la autoevaluación; ésta puede ubicarse en el paradigma de la evaluación cualitativa, ya que procura conocer el sentir y percepción de los agentes del centro, a partir de la reflexión sistemática y el diálogo profesional, con el fin de obtener la mejora del centro y el servicio que proporciona; mejorando con ello el sistema relacional, la organización y cada una las dimensiones que lo conforman.

En este orden de ideas se puede citar a González et al., (2007), cuando se refiere a la autoevaluación como "un modelo pro-activo, inductivo y cíclico que comporta un aprendizaje continuo desde la experiencia: los discursos y las prácticas de los participantes se revisan y 


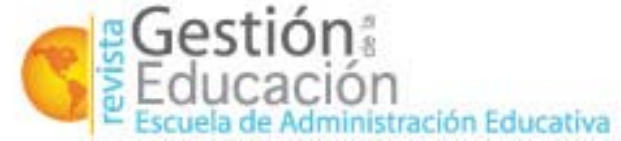

Revista Cientifica Digital ISSN-2215-2288

reconstruyen progresivamente mientras toman decisiones, emprenden acciones y se relacionan” (p.271).

\subsection{La evaluación externa}

La evaluación externa, en los centros educativos, está conformada por todas aquellas modalidades descendentes de evaluación, es decir las que se derivan de las jerarquías superiores del sistema educativo. La principal característica de la evaluación externa la constituye, haber sido diseñada por instancias superiores del sistema y por el proceso uniforme con función sumativa en su aplicación.

Señala Ruiz (2008, p. 62) que "la evaluación de los centros escolares es prescriptiva y fundamental a la hora de generar la mejora constante de la calidad de la enseñanza”. El autor plantea cuatro modelos de evaluación externa y tres alternativos. En cuanto a los modelos alternativos, presenta al modelo respondente de Stake, también denominado como sensitivo por Flórez (2003). Este modelo actúa sobre la base de los datos fenomenológicos focalizando su atención en el proceso y recibe el nombre de responsable por la forma en que incide en las acciones reales que sobre las intensiones iniciales; se lleva a cabo por quienes son los agentes de la acción.

Como segundo modelo alternativo, Ruiz (2008) describe el modelo de evaluación iluminativa de Parlett y Hamilton; su fin principal es el estudio del proyecto innovador; tiene como etapas básicas tres: observación, encuesta profundizada y explicación.

\subsection{Enfoques modélicos de evaluación institucional}

Existen diversas formas de categorizar los enfoques modélicos de evaluación institucional, según la percepción o enfoque del autor o autores; para Stufflebeam y Shinkifield (1995), citados por Mora (2004, p.11) los diferentes modelos de evaluación se agrupan de la siguiente forma: "la pseudoevaluación, la cuasievaluación, la evaluación verdadera y la evaluación holística. Las tres primeras categorías presentan los diferentes modelos desde el enfoque clásico de la evaluación.” 
Para el presente estudio, se seleccionaron tres enfoques modélicos de evaluación institucional que serán detalladamente presentados a continuación. Cada uno de ellos, desde su propia teoría, buscan el mejoramiento de la calidad de la educación que ofrece el centro que lo implementa; dos de ellos corresponden a evaluaciones externas y el tercero concierne a la evaluación interna del centro.

\subsection{Modelos de evaluación cualitativa del currículo}

De acuerdo con la literatura revisada en la evaluación del currículo, se presentan tres modelos cualitativos de evaluación curricular. El modelo sensitivo de Stake; el modelo de análisis integrado de Posner; la evaluación cualitativa a través del ojo ilustrado de Eisner. Antes de describir estos modelos evaluativos, Flórez, (2003) define currículo, en términos generales como

la manera de aplicar la teoría pedagógica en el aula a la enseñanza real. Un currículo es la mediación entre la teoría y la realidad de la enseñanza, es el plan de acción que desarrolla el profesor con sus alumnos en el aula; es una pauta ordenadora del proceso de enseñanza. (p.82)

No obstante, la anterior definición, el currículo, agrega Flórez (2003, p. 83) es mucho más, ya que implica "una concepción acerca de los contenidos, las experiencias y la actuación y secuencia para que los alumnos alcancen las metas de formación.” Considera el autor que el currículo, por sus diversas características, sólo puede evaluarse de manera cualitativa. El currículo no ha de confundirse con las metas educativas, ni con el diseño curricular, que es un proceso de difícil elaboración, cuyo producto es el mismo currículo.

\subsubsection{Modelo Sensitivo de R. Stake}

El modelo sensitivo de R. Stake, según Flórez (2003, p. 84) propone “la observación del objeto de evaluación en su ambiente natural, la consulta de las audiencias del programa para comprender las distintas percepciones que sobre él existen y la elaboración de transacciones en 


\section{Q Gégestión̊}

Revista Cientifica Digital ISSN-2215-2288

torno a esta variedad de significados sociales.” En la evaluación del currículo desde el modelo sensitivo de R.Stake, se debe identificar dentro de la organización educativa los antecedentes, es decir, las condiciones preexistentes a la interacción de los alumnos con el profesor y con el material de estudio.

\subsubsection{El modelo de análisis integrado de G. Posner}

En la aplicación de este modelo, Posner (1998), citado por Flórez (2003), propone el abordaje de la documentación del currículo oficial de la institución (del programa) de manera “directa, analítica, crítica y ecléctica, identificando los antecedentes, motivos, situaciones y circunstancias contextuales que influyeron en los diseñadores, y el peso de cada uno de los actores directamente involucrados, como la comunidad educativa, los profesores, los estudiantes y los administradores” (p.88).

Otro elemento importante a descubrir en la documentación institucional, analizada según Flórez (2003, p.88) es “el enfoque pedagógico que va a orientar la enseñanza, el concepto de formación, de aprendizaje, de enseñanza y de currículo que subyace en los materiales (...), para encontrar si la perspectiva implícita es tradicional, conductista o cognitiva. Indica Flórez (2003, p.90) que la evaluación integrada de Posner, está orientada hacia la acción, en tanto “que ilustra y propicia decisiones y acciones curriculares que requieren el mejoramiento de la enseñanza”

\subsubsection{Modelo de evaluación a través del ojo ilustrado de Eisner}

Este modelo de evaluación del currículo desde el paradigma cualitativo, hace uso de la metáfora del ojo ilustrado, la que busca comprender la cercanía del evaluador cualitativo con el objeto observado. El propósito del enfoque es conocer a partir de apreciar las cosas desde el propio yo; igualmente que como actividad intelectual, racional, en el sentido de captar, analizar e interpretar el sentido de las cosas (Flórez, 2003).

En el proceso de evaluación a través del ojo ilustrado de Eisner, de acuerdo con Flórez (2003, p.92), el evaluador observa las siguientes dimensiones: "la intencional, la estructural; la curricular, la pedagógica y la evaluativa”. Agrega Flórez (2003, p.92) que para Eisner, “ninguna escuela cambiará mientras no se cambien las prácticas evaluativas en el sentido deseado” 


\subsection{Enfoque modélico CIPP (contexto, entrada, proceso, producto)}

Flórez (2003) ubica este enfoque de evaluación en la categoría de modelo clásico, junto con el enfoque de teoría de sistemas; el modelo de evaluación CIPP aplicado a la educación se percibe como: el manejo de la información para la toma de decisiones. Stufflebeam y Shinkifield (1987), citado por Bausela, (2003, p.5), percibieron con toda claridad los componentes generales del sistema Este enfoque está conformado por cuatro etapas o fases que dirigen su aplicación “aEvaluación del contexto; b- evaluación de entrada; c- evaluación del proceso; y d- evaluación del producto."

Considera Martínez (1996), citado por Bausela, (2003), que en el enfoque CIPP los tipos de decisión que se deben enfrentar en la organización están asociados a los tipos de evaluación institucional. La evaluación del contexto nos proporciona información para las decisiones de planificación, con el fin de determinar los objetivos. La evaluación de entrada, nos proporciona información para establecer decisiones de tipo estructural y de procedimiento con el fin de seleccionar el diseño de un programa. La evaluación del proceso nos proporciona información para las decisiones de implementación con el fin de aceptar, clarificar o corregir el diseño tal como realmente se ha llevado a cabo. La evaluación del producto nos proporciona información para tomar decisiones de reciclaje con el fin de aceptar, rectificar o abandonar el programa.

\subsection{La autoevaluación como enfoque de evaluación institucional}

Como enfoque de evaluación interna, la autoevaluación presenta también algunas dimensiones o ámbitos en donde se acciona la práctica evaluativa. El Modelo de autoevaluación interna presentado por Antúnez y Carnicero (2009), definen los siguientes ámbitos o dimensiones objeto de análisis en una evaluación institucional:

- Académico

- Administrativo

- Organizativo

- Sistema de relaciones

- Programas proyectos y servicios complementarios (pp. 130-137) 
Recomienda Antúnez y Carnicero (2009, p. 138) que “el proceso de autoevaluación se realice una vez al año, en sus fases de sensibilización, planificación, diagnóstico, interpretación y propuesta de plan de mejora desarrollándolo en las últimas semanas del año”. Sin dejar pasar las tareas de revisión procesal, dando espacio para el análisis de los resultados en la implementación de la propuesta de mejora a realizar durante las mismas.

La autoevaluación como modelo de evaluación interna según, Ruiz (2008, p. 30), es “una práctica que todavía no está en la concepción del trabajo colectivo en los centros escolares, cada profesor actúa en su aula y se preocupa casi en exclusiva, en términos evaluativos, de la evaluación/valoración del aprendizaje de sus alumnos.” La autoevaluación en su amplia concepción requiere de tiempo, disposición, motivación y compromiso por parte del equipo docente que participa en ella y los directivos de la organización.

\subsection{La autoevaluación como proceso de mejora educativa}

El cambio es una de las razones de ser de la evaluación de un centro educativo. En este sentido De Miguel y otros (1994), citado por Escudero (1997), consideran la evaluación necesaria para el cambio, fundamental para las escuelas eficaces, lo que ha generado un movimiento entorno a la mejora de la escuela, orientado a la búsqueda de aspectos del centro educativo que inciden en su funcionamiento y eficacia orientado hacia lo que se debe mejorar y del modo en que deben hacerse. Para Escudero (1997) cualquier modelo de evaluación tiene su incidencia según corresponda en la mejora del centro educativo, y esta mejora debe ser un objetivo de todo modelo de evaluación en los centros educativos.

No se trata de definir un modelo para alcanzar la mejora del centro, sino que a partir del modelo que se utilice, este debe tener como fin el alcanzar la mejora institucional. Para ello, indica Escudero (1997) que se debe diagnosticar los problemas de la organización y determinar los recursos con que se cuenta para resolverlos. Además, que para realizar este proceso de diagnosticar los problemas institucionales, la autoevaluación cobra relevancia por fomentar los procesos de participación y de reflexión por parte de quienes forman parte del centro, así como el uso responsable y colegiado de la autonomía del centro. 
Según Santos (1995) la autoevaluación procura dar respuesta a las siguientes interrogantes: ¿Qué está sucediendo aquí?, ¿Estamos haciendo lo que nos proponemos?, ¿Estamos consiguiendo lo que buscamos?, ¿Estamos consiguiendo precisamente lo contrario? ¿Por qué? La autoevaluación en el centro, es necesaria por la capacidad que tiene para el desarrollo curricular y para la mejora de la profesionalidad de los docentes.

Corresponde al equipo directivo, impulsar la apertura de los espacios para la reflexión permanente y compartida sobre la acción educativa. Santos (1995, p. 141) propone una evaluación que "nace de la iniciativa del Centro, se realiza por los mismos profesionales que ejercen la enseñanza y tiene por objetos comprender y mejorar la práctica educativa.” La autoevaluación es una acción por iniciativa de la gestión directiva y con la participación plena y comprometida por parte del cuerpo docente y el resto de la comunidad educativa.

\subsection{La evaluación institucional como gestión de la educación}

En el marco de una política de empoderamiento del centro educativo como eje vertebrador del mejoramiento de la calidad de la educación, el Ministerio de Educación Pública de Costa Rica [MEP] (2008), junto con el Consejo Superior de Educación, lo propone como respuesta al proceso de reflexión para lograr un Acuerdo Nacional sobre Educación, con el propósito de nutrir con sus resultados la construcción conjunta de una Política de Estado en Educación y con ello, responder a la sociedad costarricense.

La gestión educativa de todo centro educativo está llamada a orientar su acción en la consecución de un proyecto nacional hacia la mejora institucional educativa, en donde la evaluación desempeñará un rol trascendental para alcanzar los objetivos fijados en este proceso de mejora en la calidad de la educación que brindan a la población educativa que la conforma. A la gestión educativa del centro educativo le corresponde asegurar que se cumplan los fines de la educación, esto como parte de sus funciones.

La autoevaluación, expresa Escudero (1997), evidencia la capacidad de cada organización para autotransformarse. En efecto, el centro educativo que busca la mejora de la calidad de la enseñanza y la innovación, encontrará en el proceso de autoevaluación, la herramienta que le posibilitará tan importante transformación. 


\subsection{Incidencia de los enfoques de evaluación en la gestión de los centros educativos}

Los modelos de evaluación institucional, sin importar su fuente, ya sea interna o externa, o su paradigma cuantitativo o cualitativo, tienen como fin generar a lo interno del centro, la información necesaria que será utilizada para llevar a cabo los cambios pertinentes que conlleven a la mejora del servicio ofrecido, se considera también, el poder de mejoramiento hacia el equipo docente, administrativo y con mayor incidencia hacia la comunidad estudiantil.

Para Antúnez y Carnicero (2009) la promoción y desarrollo de innovaciones en los centros escolares a partir de la evaluación institucional, son parte del mejoramiento de la calidad de la educación, no obstante puede encontrar obstáculos en la resistencia a los cambios por parte de las personas; es por ello, que es de suma importancia que la gestión educativa impulse las fases de comunicación, sensibilización, motivación y justificación que deben preceder a un proceso de evaluación institucional. El principal valor agregado de la evaluación institucional, lo constituye el crecimiento profesional de los agentes docentes, el mejoramiento en la calidad de la enseñanza que se proporciona, en la capacidad de gestión de los directivos del centro, en la calidad de las decisiones por tomar, en el mejoramiento del clima organizacional o relacional.

\section{Referente metodológico}

El trabajo de investigación realizado se enmarca, por su carácter y profundidad, dentro del enfoque cuantitativo descriptivo, ya que busca definir un fenómeno, así como especificar las características y componentes del enfoque de evaluación institucional aplicado por la gestión administrativa en una comunidad educativa. Para efecto del trabajo de investigación, la población está conformada por el personal docente, docente administrativo, técnico docente, que forma parte de la organización educativa Costa Rica Christian School [CRCS] para el curso lectivo del 2010. Un total de 42 funcionarios participaron en el estudio.

Para recolectar la información se elaboró un cuestionario semi- estructurado, conformado de tres partes, el cual se auto administró en el centro educativo. El cuestionario busca dar respuesta a los objetivos específicos, a partir de la definición de las categorías de análisis; en donde los indicadores fueron organizados de manera paralela a los enfoques considerados para el estudio, iniciando con el enfoque de evaluación institucional de evaluación del currículo; así 
mismo la definición instrumental inicia de manera paralela a la definición operacional de cada enfoque modélico.

La primera parte del cuestionario responde a la identificación del enfoque de evaluación institucional aplicado por la gestión de un centro educativo privado, a través de las principales aspectos que caracterizan el enfoque, para lo cual se presentó un total de 64 ítems de preguntas cerradas y dos abiertas, distribuidos en los tres enfoques modélicos, en donde el modelo de autoevaluación presenta la mayor parte de los ítems. Ésta primera parte del cuestionario tiene una estructura que combina las respuestas cerradas de SI o NO y una escala de Likert de tres opciones del $1 \mathrm{al}$ 3, siendo el tres el valor de siempre, 2 casi siempre y 1 nunca. La respuesta SI o No responde a la presencia o no de un enunciado, en caso afirmativo, la frecuencia con que éste se presenta en el quehacer del proceso evaluativo de la institución.

Una vez identificado el enfoque de evaluación, la segunda parte del cuestionario está dirigida a determinar las dimensiones presentes en el enfoque de evaluación institucional identificado por la población participante, para esta sección del cuestionario se destinaron 16 ítems de respuesta cerrada (si o no) y cuatro preguntas abiertas. En este apartado se presentaron las dimensiones que integran los tres enfoques propuestos, entre los que se puede citar: el recurso humano, el currículo institucional, el entorno y herramientas tecnológicas, el proceso de evaluación de los aprendizajes, las necesidades de la comunidad estudiantil y la evaluación de la evaluación institucional (metaevaluación) como dimensiones del enfoque de evaluación del currículo. Para el segundo enfoque (CIPP), las dimensiones presentadas tienen que ver con: el entorno institucional, los programas institucionales, los recursos materiales, el currículo institucional. El tercer enfoque correspondiente a la autoevaluación, presenta las dimensiones relacionadas con: el área académica y sus necesidades, el área administrativa, el área organizativa, el sistema de relaciones existente y por último la dimensión de los programas, proyectos y servicios. Cada una de las dimensiones está fundamentada en la referencia teórica que sustenta el estudio.

La tercera parte del cuestionario se formuló con el objetivo de dar respuesta al tercer objetivo específico, que responde a determinar la percepción que el enfoque de evaluación institucional aplicado por la gestión educativa de Costa Rica Christian School [CRCS] ha 
desarrollado entre el personal de la organización educativa. Para lo anterior se presentó una serie de diez preguntas cerradas, en lo relacionado a: la toma de decisiones que favorecen el cambio y mejora de la organización, el diseño y mejoramiento del proyecto educativo, implementación de planes de desarrollo profesional para el personal, la mejorara del servicio que se ofrece, mejoras en el ambiente relacional de la organización, la atención de las necesidades emergentes de manera pronta eficaz y eficiente, la promoción del diseño de proyectos de innovación, y por último la participación del personal de la organización de manera activa en el diseño del enfoque de evaluación institucional.

Las fuentes de información se conformaron de la siguiente manera: Fuentes Primarias, integradas por los y las docentes de los diferentes ciclos, así como el personal docente administrativo; el personal técnico-administrativo y administrativo de la organización. Fuentes secundarias, la conforman los textos de la revisión bibliográfica, artículos, nacionales e internacionales en forma física y digital, así como trabajos finales de graduación igualmente a nivel nacional e internacional, consultados para tal fin.

Para la validación del instrumento, se aplicó el mismo a un grupo de profesionales en educación quienes realizaron la devolución sobre los aspectos que consideraron propicios o no del cuestionario en cuanto a vocabulario, temática, extensión y tiempo.

\section{Sistematización y análisis de la información}

La sistematización y análisis de los principales resultados del estudio se orienta según los objetivos de la investigación: identificación del enfoque de evaluación institucional aplicado por la gestión educativa en Costa Rica Christian School [CRCS]; la determinación de las dimensiones que conforman el enfoque de evaluación institucional aplicado por la gestión educativa en CRCS; y la determinación de la percepción que el enfoque de evaluación institucional aplicado por la gestión educativa de CRCS ha desarrollado entre el personal de la organización educativa en el funcionamiento del centro escolar. 


\subsection{Análisis del enfoque modélico de evaluación aplicado por la gestión educativa en Costa Rica Christian School}

En el estudio se utilizó el referente teórico de tres enfoques modélicos de evaluación institucional con sus respectivas características y dimensiones. Los siguientes son los enfoques que sirvieron de sustento teórico a la investigación: el enfoque de evaluación del currículo, el enfoque contexto, entrada, proceso y producto (CIPP) y el enfoque de autoevaluación. El análisis de cada enfoque se realizó de manera independiente.

\subsection{Análisis de las características del enfoque de evaluación del currículo}

El enfoque de evaluación del currículo para efecto del estudio presenta seis características, las que están dirigidas a evaluar dimensiones como: el recurso humano, el propio currículo institucional, el entorno y las herramientas tecnológicas, el proceso de evaluación de los aprendizajes; las necesidades de la comunidad estudiantil y el proceso de evaluación de la evaluación, es decir, la metaevaluación institucional.

La identificación del enfoque de evaluación del currículo, a partir de las características del mismo, es importante para el estudio ya que, se considera que identificando los elementos que lo caracterizan, se puede determinar el tipo de modelo de evaluación aplicado por la gestión educativa de la organización.

Como resultado del estudio, en la identificación de las características del primer enfoque modélico de evaluación institucional, el personal docente de CRCS, consideró en un 76\% que en la organización se toman en cuenta dichas características como parte del enfoque aplicado por la gestión educativa. Sin embargo, la falta de una respuesta categórica en relación a la identificación, advierte un serio problema a lo interno de la organización en cuanto a la aplicación de un enfoque de evaluación institucional para todos los miembros del personal. 


\section{Girgestión:}

Revista Cientifica Digital ISSN-2215-2288

\subsection{Análisis de las características del enfoque modélico de evaluación CIPP}

El segundo enfoque modélico de evaluación institucional que se analiza en el estudio corresponde al enfoque de Evaluación CIPP (contexto, entrada, proceso y producto) de Stufflebeam. Los resultados en esta etapa de la investigación, no alcanzaron el mínimo establecido como positivos para considerar las características de este enfoque como parte un proceso de evaluación institucional, aplicado por la gestión educativa en la organización. El gráfico \# 1 resume las seis características de la fase de producto del enfoque CIPP, se puede observar de manera general la percepción cuantitativa que sobre esta fase manifestó el personal del centro, solamente el 39\% expresó que estas características son aplicadas por parte de la gestión educativa de CRCS.

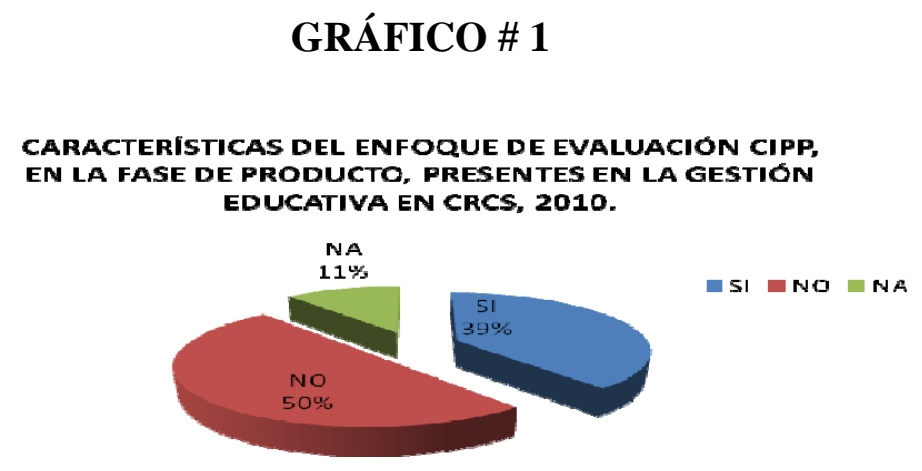

Fuente: Cuestionario aplicado a los docentes de CRCS, 2010.

En síntesis, las once características que en el estudio se tomaron en cuenta para representar las cuatro fases del enfoque de evaluación institucional CIPP, no reflejaron en los resultados estar presentes en la organización como parte del enfoque de evaluación institucional aplicado en la organización. El estudio descarta tanto, la presencia del enfoque de evaluación del currículo, así como el enfoque CIPP, como parte del proceso de evaluación institucional aplicado en la organización. 


\subsection{Análisis de las características del enfoque de evaluación institucional autoevaluación}

De acuerdo con el ordenamiento que se ha venido realizando y, como parte del análisis para identificar el enfoque de evaluación institucional aplicado por la gestión educativa; se presentó un tercer enfoque en el estudio, correspondiente al enfoque de autoevaluación. La información del mismo, se analizó según cada una de sus dimensiones, tal y como fue presentado en el cuestionario al personal que participó en el estudio. Las caracterísitcas están representadas en las dimensiones que desarrollada el instrumento a saber: la académica; la administrativa; la organizativa; sistema de relaciones y por último pero, no menos importante, la correspondiente a programas, proyectos y servicios complementarios.

Para la dimensión académica de manera general, el 75\% del personal participante consideró que la gestión educativa de CRCS, aplica las características correspondientes a la dimensión académica del enfoque de la autoevaluación como parte del proceso de evaluación institucional. Sin embargo tampoco se observa una condición unánime que refleje conocimiento pleno del enfoque que emplea la gestión educativa.

La percepción, del personal participante, en la identificación de las características correspondientes al enfoque de autoevaluación en el ámbito administrativo, alcanza un 67,89\% del reconocimiento de éste como parte del enfoque de evaluación aplicado por la gestión educativa de la organización, no obstante, este porcentaje no es suficiente para identificarlo, en su ámbito administrativo, como un enfoque sistemático de evaluación aplicado en la institución. No hay claridad en el personal para idenficar el enfoque de autoevalaución en sus características administrativas.

Las características correspondientes al enfoque de evaluación institucional de autoevaluación, en su ámbito organizativo, es identificado por el personal participante en un 72\%, como características del enfoque de evaluación institucional aplicado por la gestión educativa de CRCS. Existe un porcentaje (23\%) que no considera presente esta dimensión y otro que indica que no aplica (9\%).

En cuanto a la existencia de las características del sistema de relaciones, es claro entre el personal de la organización, las normas de convivencia, los principios de justicia, equidad, transparencia y respeto, así como normas para un servicio de calidad, están presentes en la 
organización, lo que favorece el ambiente laboral en lo relacional entre los profesionales y los estudiantes. Aspecto importante en que la gestión educativa debe intervenir de manera intencional. Esta es una de las características presentes en el estudio que alcanzaron un rango superior al $80 \%$, solo un $4 \%$ consideró que no aplicaba para ellos.

En la última dimensión del enfoque de autoevaluación, el personal participante solo considera en un 54\% que la gestión educativa aplica estas características del enfoque, en la organización; porcentaje que no es sufienciente como considerarlo parte de un enfoque de evaluación institucional en CRCS. Es importante considerar que un alto porcentaje, del personal participante, un $24 \%$, indica que no aplica o no tiene conocimiento de las características que forman parte de un proceso de evaluación institucional en el enfoque de la autoevaluación.

A manera de colofón de esta primera etapa del análisis, se puede advertir que, a pesar de la existencia en la organización de algunas de las características que distinguen a los enfoques expuestos en el estudio, la identificación de un enfoque a partir de ellas, no fue posible por parte del personal participante, lo que evidencia la falta de claridad en los procesos de evaluación institucional que posee el personal a partir de la gestión educativa.

\subsection{Análisis de las dimensiones presentes en el enfoque de evaluación institucional aplicado por la gestión educativa en Costa Rica Christian School}

El siguiente esquema presenta las dimensiones del enfoque de evaluación del currículo de manera específica. 


\section{Qis:Gestión

\section{ESQUEMA 1 \\ DIMENSIONES DEL ENFOQUE DE EVALUACIÓN DEL CURRÍCULO}

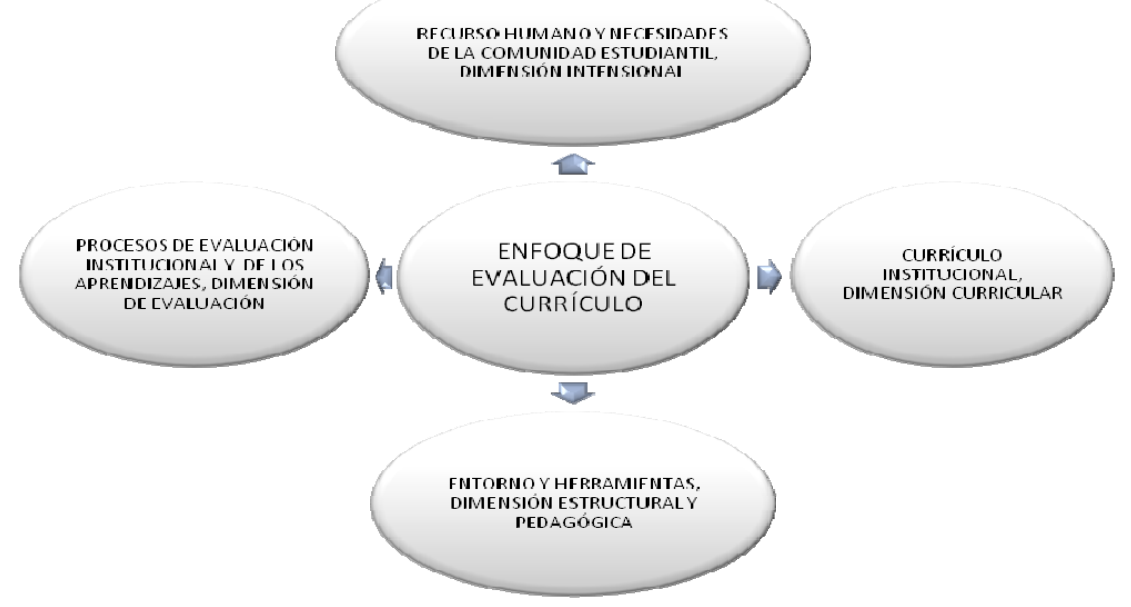

Fuente: Estudio elaborado por Ana Jenssie Campos C. sobre el enfoque modélico de evaluación institucional aplicado por la Gestión Educativa en CRCS, 2010.

La percepción del personal que participó en el estudio, en cuanto a la determinación de las dimensiones del enfoque de evaluación del currículo presentes como enfoque de evaluación institucional que aplica la gestión educativa en CRCS; muestra que solo el 33\% de los y las participantes en el estudio identifican las dimensiones correspondientes al enfoque de evaluación del currículo.

Por los resultados obtenidos se puede afirmar que para el personal participante en el estudio, las dimensiones del enfoque de evaluación del currículo no están presentes como enfoque de evaluación institucional aplicado por la gestión educativa de Costa Rica Christian School [CRCS]. Lo anterior, coincide con la percepción que el personal participante establece sobre la identificación de las características para éste enfoque.

\subsection{Análisis de las dimensiones del enfoque de evaluación institucional CIPP}

En relación al enfoque de evaluación CIPP, como enfoque de evaluación institucional externa; al tomar en cuenta diferentes aspectos de la evaluación institucional de manera integral 


\section{Q Gestión:}

lo posiciona como un enfoque útil para el análisis de la organización; y a partir de los resultados, orientar la toma de decisiones que debe alcanzarse, en función de la mejora en la planificación institucional.

\section{ESQUEMA \# 2 \\ DIMENSIONES DEL ENFOQUE DE EVALUACIÓN CIPP}

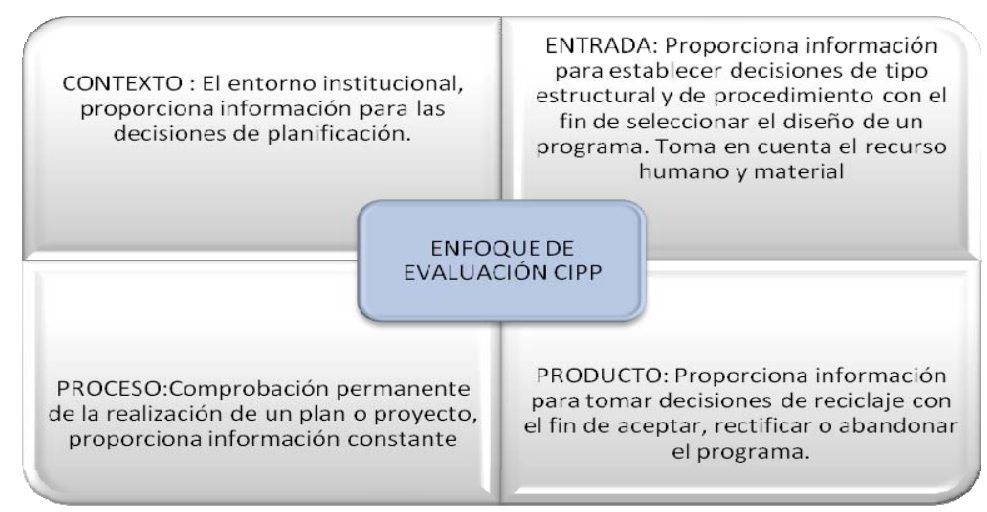

Fuente: Estudio elaborado por Ana Jenssie Campos C. sobre el enfoque modélico de evaluación institucional aplicado por la Gestión Educativa en CRCS, 2010.

Como resultado del análisis de este enfoque en cuanto a la presencia de sus dimensiones en el proceso de evaluación institucional, solo el 47\% del personal determina que las dimensiones de este enfoque están presentes en la organización; es importante indicar que el 24\% no dio respuesta en el instrumento a este aspecto, lo que advierte que el personal participante no está seguro sobre las dimensiones que componen y están presentes en el enfoque de evaluación aplicado por la gestión educativa del centro en estudio. Otro porcentaje significativo es el que respondió en un 27\%, que las dimensiones del enfoque CIPP no están presentes como parte del enfoque de evaluación institucional.

\subsection{Análisis de las dimensiones del enfoque de autoevaluación}

El tercer y último enfoque de evaluación institucional a determinar sí sus dimensiones están presentes en el proceso de evaluación institucional aplicado por la gestión educativa, es el denominado autoevaluación. La autoevaluación como modelo de evaluación interna, según Ruiz 


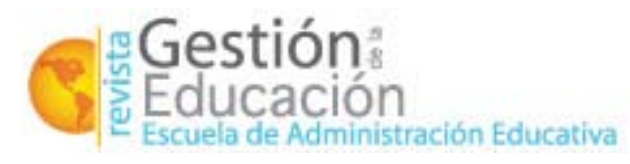

Revista Cientifica Digital ISSN-2215-2288

(2008, p. 30) es “una práctica que todavía no está en la concepción del trabajo colectivo en los centros escolares, cada profesor actúa en su aula y se preocupa casi en exclusiva, en términos evaluativos, de la evaluación/valoración del aprendizaje de sus alumnos.” Es común que en nuestros centros de educación el énfasis de la evaluación se realice alrededor del desempeño del estudiante.

En la realización del estudio, se consideró el Modelo Regional de Gestión Escolar en Centroamérica y República Dominicana, el cual, presenta el modelo de autoevaluación como modelo de evaluación interna por seguir en el Área, según Antúnez y Carnicero (2009) sus dimensiones o ámbitos son: a- Académico; b- Administrativo; c- Organizativo; d- Sistema de Relaciones; e- Programas, Proyectos y Servicios Complementarios. Como se presenta en el siguiente esquema:

\section{ESQUEMA 3 \\ DIMENSIONES DEL ENFOQUE DE AUTOEVALUACIÓN}

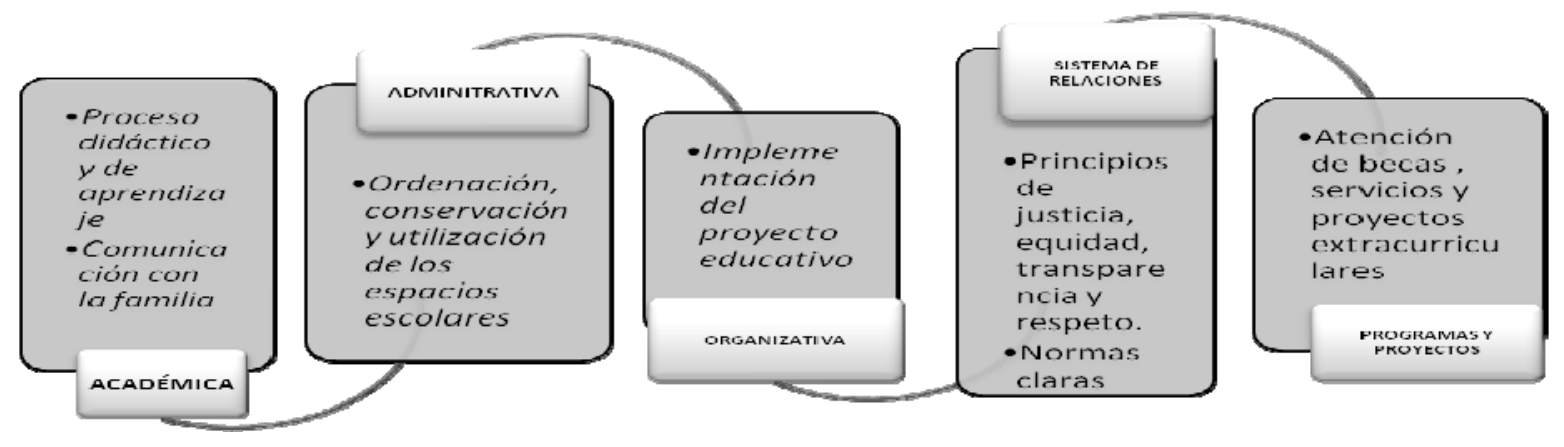

Fuente: Estudio elaborado por Ana Jenssie Campos C. sobre el enfoque modélico de evaluación institucional aplicado por la Gestión Educativa en CRCS, 2010.

En el estudio, al determinar que dimensiones del enfoque modélico de la autoevaluación están presentes en el proceso de evaluación aplicado por la gestión educativa de CRCS, el personal participante en un $11 \%$ considera la presencia de dichas dimensiones como parte del enfoque de evaluación institucional, por otra parte, el 75\% no logra identificar las dimensiones correspondientes al enfoque de autoevaluación, lo que conlleva a concluir que las dimensiones del enfoque modélico de autoevaluación como evaluación institucional interna, no está definido en la organización. 
De acuedo con la investigación, el 64\% del personal considera que no existe un enfoque claro de evaluación institucional, y el 36\% del personal optaron por no responder o indicaron que no aplica en el cuestionamiento. Esta percepción es concluyente para determinar la presencia de las dimensiones que conforman los diferentes enfoques de evaluación, en el proceso de evaluación institucional aplicado por la gestión educativa .

En el análisis de los tres enfoques modélicos propuestos para la investigación, aunque se identificaron algunas características, de cada uno así como, algunas de sus dimensiones, éstas se dieron de manera parcial; por lo que no es posible determinar de manera categórica la aplicación de un enfoque modélico determinado, por parte de la gestión educativa del centro.

\subsection{Análisis de la percepción del personal acerca del enfoque de evaluación institucional}

El Ministerio de Educación Pública [MEP] (2008, p. 21) hace referencia a que la gestión educativa debe estar en función del proceso educativo, en donde la "evaluación integral permanente y, consecuentemente, la introducción de las acciones correctivas que permitan el logro de los objetivos esenciales programados”. La sociedad, a través de las estructuras políticas, demanda de la gestión educativa, apoyar la educación de manera ágil, eficiente y amable. Es un reto cuyo alcance, está dirigido a transformar no solo el centro educativo, sino el sistema en general.

Como resultado del análisis del tercer objetivo, en relación a la determinación de la percepción que el enfoque de evaluación institucional aplicado por la gestión educativa de CRCS ha desarrollado entre el personal de la organización; solo el 23\% del personal participante, considera que existe algún nivel de influencia del enfoque de evaluación institucional aplicado por la gestión educativa en CRCS, un 39 \% considera que el enfoque de evaluación aplicado por la gestión educativa no ejerce influencia en los diferentes aspectos de la organización del centro. Además, puede observarse que el 35\% indicó que ese aspecto en el cuestionamiento no aplica, es decir, se abstuvo de dar una respuesta positiva o negativa lo que en realidad llega a fortalecer la percepción negativa acerca de la influencia que puede ejercer sobre la organización el enfoque aplicado por la gestión educativa del Centro en estudio. 
La participación directa de la gestión educativa en el desarrollo de una cultura institucional de evaluación, permite el impulso de un proceso de toma de decisiones de una manera participativa, favoreciendo en la organización el diseño y mejoramiento del proyecto educativo, éstos son elementos que el personal participante considera no se están presentando en la organización; ésta condición eventualmente no permitirá que la organización avance en la transformación educativa que demanda la sociedad actual.

\section{Conclusiones}

La identificación del enfoque modélico aplicado por la gestión educativa en Costa Ria Christian School [CRCS], no fue claro y concluyente para ninguno de los tres enfoques presentados en el estudio, más parece una combinación de enfoques que reúne una variedad de características, que un enfoque sistemático de evaluación institucional, lo que conlleva a una desventaja en la detección oportuna de necesidades y la atención de las mismas en forma eficiente y eficaz, de manera que se asegure la mejora en la calidad del proyecto educativo de la organización.

La determinación de los ámbitos o dimensiones, asociados a los enfoques modélicos de evaluación institucional están estrechamente relacionados con la identificación de los mismos; en el estudio, el personal participante divide su criterio de manera marcada, al momento de determinar las dimensiones presentes, sin embargo, entre los tres enfoques modélicos al igual que con la identificación de la características, no existe claridad por parte del personal participante, para determinar las dimensiones correspondientes a un enfoque de evaluación institucional que se aplique en la organización por parte de la Gestión Educativa de forma terminante; por lo anterior, se puede afirmar que no existe en la organización educativa un enfoque modélico sistemático de evaluación institucional.

En cuanto a la percepción que el personal de la organización tiene acerca de la influencia que sobre el funcionamiento del centro escolar, tiene la aplicación de un enfoque de evaluación institucional determinado, que conduzca a la transformación de la organización a través de un plan de mejora, no llega a ser efectivo o influyente; por lo que conlleva a una gestión educativa 


\section{Gestión:}

Revista Cientifica Digital ISSN-2215-2288

poco eficiente en cada uno de los aspectos que se consideran importantes para el mejoramiento en la calidad de la educación; como lo es la toma de decisiones efectivas y, eficaces, la innovación, y el crecimiento profesional, lo anterior desfavorece el proyecto educativo en tanto no se atienden las necesidades o no se reconocen ni se priorizan;

Después de lo expuesto, se hace necesaria la intervención inmediata en cuanto a la temática de evaluación institucional a través de la capacitación de personal y selección de un enfoque modélico que guíe la evaluación interna del centro, o bien se determine una evaluación externa que contribuya igualmente, a la mejora de la calidad de la educación que se ofrece a la comunidad educativa que atiende.

La administración de la educación ante el reto de liderar la organización educativa de manera eficaz y eficiente, puede encontrar en la evaluación institucional, el mejor aliado como herramienta para alcanzar los cambios que requiere el centro, garantizando una educación que forma los ciudadanos del futuro, capaces de enfrentar los desafíos que le presenta la sociedad del conocimiento, en donde las nuevas tecnologías son más complejas y requiere de respuestas prontas, oportunas y eficaces.

A continuación se presenta una síntesis de la propuesta de intervención desarrollada para este estudio.

\section{Evaluación Institucional del Centro a partir del Enfoque Modélico de Evaluación Interna “Autoevaluación”}

La evaluación institucional tiene como una de sus finalidades promover el cambio e innovación en el servicio educativo que se ofrece. Esta transformación busca el mejoramiento en la calidad de la educación que recibe la generación actual. La gestión educativa como parte de sus funciones tiene esta responsabilidad, de tal manera que, dé respuesta a la sociedad actual, que demanda nuevas competencias para asumir con responsabilidad los nuevos retos sociales y profesionales.

Como parte de la responsabilidad de los gestores de la educación Garbanzo y Orozco (2007, pp.101-102) indican que "La calidad de la educación es una responsabilidad compartida, 
donde los gestores de la educación deben tener la capacidad de trasmitirla y concebirla, no solo como un producto, sino como un proceso por el que se lucha.” Además, "la gestión educativa de calidad es responsabilidad de los actores sociales involucrados en la educación, lo cual incluye la sociedad civil en su totalidad.” En otras palabras, no se trata de una tarea exclusiva de la gestión educativa, es un compromiso de la sociedad a través de los diferentes actores sociales que interactúan en la comunidad en donde se encuentra inmerso el centro.

La evaluación institucional, como proceso de evaluación interno o externo, permite a la gestión educativa valorar aquellas dimensiones en las que considere necesario determinar necesidades que requieran de la toma de decisiones que conduzcan a mejorar el servicio ofrecido, una vez que se identifican y se toman en cuenta en la planificación institucional.

Con base en las observaciones anteriores, se presenta una propuesta de un proyecto de autoevaluación institucional, que pretende ofrecer a la administración de la educación del centro, herramientas en el campo de la evaluación institucional, de esta forma coadyuvar con los fines y objetivos de la educación.

El diseño está orientado en dos etapas importantes en la autoevaluación: fomento de una cultura evaluativa interna de centro y la implementación de un proyecto institucional de autoevaluación. La siguiente figura muestra las dos etapas por desarrollar en la propuesta.

\section{Etapas de intervención del proyecto de autoevaluación institucional}

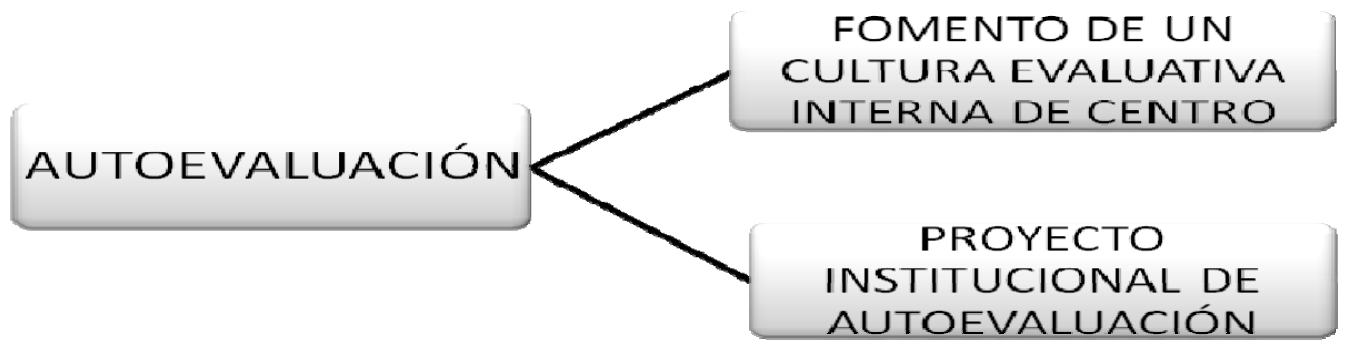

Fuente: Estudio elaborado por Ana Jenssie Campos C. sobre el enfoque modélico de evaluación institucional aplicado por la Gestión Educativa en CRCS. 


\subsection{Desarrollo de la Propuesta}

\subsubsection{Acciones del proyecto de autoevaluación como enfoque de evaluación institucional.}

Considerando los aspectos implícitos, dentro de cada una de las áreas por intervenir en el proyecto de autoevaluación, como evaluación institucional, se presentan acciones que permitan a los agentes sociales del centro llevar a cabo, el proceso de manera que se favorezca la toma de decisiones en el interior de la organización y con ello la mejora institucional.

\subsubsection{Fomento de un cultura evaluativa}

Esta etapa comprende todas aquellas acciones que conlleven al establecimiento de una cultura evaluativa dentro de la organización.

\subsubsection{Definición de las etapas que forman parte del Proyecto de Autoevaluación}

Esta etapa comprende todas aquellas acciones que se han de llevar a cabo para desarrollar las etapas que forman parte de un proyecto de autoevaluación institucional, según el Ministerio de Educación Pública [MEP] (2008).

\subsection{Objetivos de la propuesta}

\subsubsection{Objetivo General:}

- Diseñar un proyecto de “autoevaluación”, como evaluación institucional, viable para ser aplicado por la Gestión Educativa del “Costa Rica Christian School”. 


\subsubsection{Objetivos Específicos:}

- Establecer una cultura de autoevaluación institucional entre los miembros de la organización educativa.

- Determinar los pasos por seguir para llevar a cabo el proyecto de autoevaluación a partir de la definición de dimensiones del proyecto de autoevaluación.

\subsection{Plan de Acción}

Para el desarrollo del proyecto de autoevaluación se proponen las siguientes temáticas: fomento de una cultura evaluativa y etapas del proyecto de autoevaluación. Los siguientes esquemas presentan las etapas a implementar.

\subsubsection{Fomento de una cultura evaluativa:}

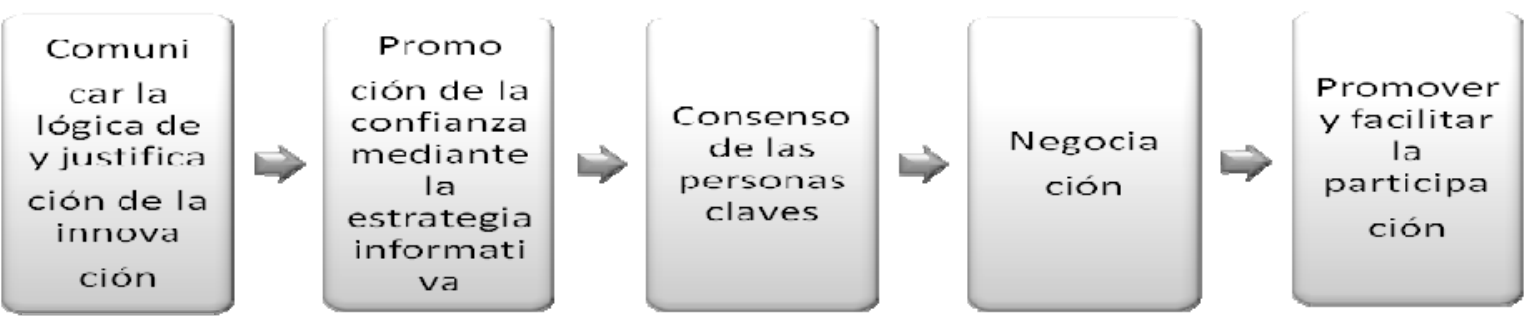

\subsubsection{Etapas del proyecto de autoevaluación:}




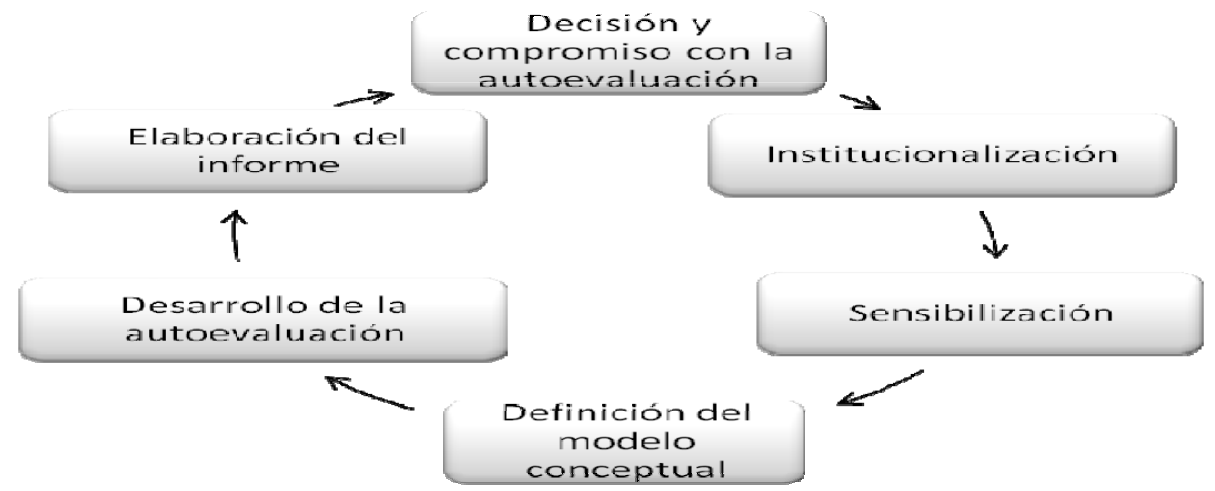

Considerando que la gestión educativa es quien tiene la responsabilidad de promover a lo interno de la organización, los procesos necesarios para llevar a cabo las acciones que conlleven a la mejora de la calidad de la educación y uno de los procesos más importantes corresponde a la evaluación institucional, se hace imprescindible una gestión participativa que facilite la promoción de un enfoque de evaluación acorde con las necesidades de la organización, la construcción de una cultura de autoevaluación en nuestros centros educativos se constituye en un reto para toda gestión educativa que busque la mejora de la calidad de la educación en nuestra sociedad.

\section{Referencias Bibliográficas}

Antúnez, S. y Carnicero, P. (2009). Modelo Regional de Gestión Escolar en Centroamérica y República Dominicana: Análisis de la situación y propuestas para la Convergencia Regional. República Dominicana: Colección IDER.

Bausela, E. (2003). Metodología de la Investigación Evaluativa: Modelo CIPP1. Revista Complutense de Educación, 14(2), 361-376. Universidad de León. Recuperado de http://revistas.ucm.es/edu/11302496/articulos/RCED0303220361A

Delors, J., Mufti, I., Amagi, I., Carneiro, R., Chung, F., Geremek, B. y Nanzhao, Z. (1996). La Educación Encierra un Tesoro. Informe a la UNESCO de la Comisión Internacional sobre la Educación para el siglo XXI (Compendio). Francia: Santillana Ediciones UNESCO.

Edwards, R. V. (1991). El concepto de calidad de la educación. Oficina Regional de Educación de la UNESCO para América Latina y el Caribe. Santiago, Chile: OREAL/UNESCO. 
Escudero, T. (1997). Enfoques modélicos y estrategias en la evaluación de centros educativos. Revista Electrónica de Investigación y Evaluación Educativa [RELIEVE], 1(3). Recuperado de http://www.uv.es/RELIEVE/v3n1/RELIEVEv3n1_1.htm

Flórez, O. R. (2003). Evaluación Pedagógica y Cognición. Colombia: McGraw.Hill Interamericana, S.A.

Garbanzo V., G. y Orozco D., V. (2007). Desafíos del Sistema Educativo Costarricense: Un nuevo paradigma de la administración de la educación. Revista Educación, 31 (2). Recuperado de http://www.latindex.ucr.ac.cr/educacion-31-2/06-GARBANZO.pdf

González, G. T. Nieto, C. J. y Portela, P. A. (2007). Organización y Gestión de Centros Escolares: Dimensiones y Procesos. Madrid: Pearson-Prince Hall.

Ministerio de Educación Pública [MEP]. (2008). El Centro Educativo de Calidad como Eje de la Educación Costarricense. San José, Costa Rica: MEP.

Mora V., A. I. (2004). La Evaluación Educativa: Concepto, Períodos y Modelos. Revista Actualidades Investigativas en Educación, 4(2), 1-28. Instituto de Investigación en Educación [INIE]. Universidad de Costa Rica. Recuperado de http://revista.inie.ucr.ac.cr/articulos/2-2004/archivos/periodos.pdf

Ruiz, J.M. (2008). Cómo hacer una Evaluación de Centros Educativos. Madrid: Narcea, S.A.

Santos, G. M. (1995). La Evaluación: un Proceso de Diálogo, Compresión y Mejora. España: ALJIBE. 